\title{
Idiopathic Bronchiolocentric Interstitial Pneumonia
}

Samuel A. Yousem, MD, Sanja Dacic, MD, PhD

Department of Pathology, University of Pittsburgh Medical Center, Presbyterian University Hospital, Pittsburgh, Pennsylvania

The authors report 10 patients with a distinctive idiopathic bronchiolocentric interstitial pneumonia having some histologic similarities to hypersensitivity pneumonitis. Bronchiolocentric interstitial pneumonia has a marked predilection for women $(80 \%)$ and occurs in middle age (40-50 years). Chest radiographs and pulmonary function tests show interstitial and restrictive lung disease, while the histologic appearance is that of a centrilobular inflammatory process with small airway fibrosis and inflammation that radiates into the interstitium of the distal acinus in a patchy fashion. Granulomas are not identified. At a mean followup of approximately 4 years in nine patients, $33 \%$ of patients were dead of disease and $56 \%$ had persistent or progressive disease suggesting a more aggressive course than hypersensitivity pneumonitis and nonspecific interstitial pneumonia, the two major disease processes in the differential diagnosis. Whether Bronchiolocentric interstitial pneumonia is a unique entity or not, the pattern of bronchiocentric injury to the lung in the absence of known causes and its clinical presentation as interstitial lung disease, warrants further investigation of this unusual interstitial process.

KEY WORDS: Bronchiolitis obliterans, Hypersensitivity pneumonitis, Nonspecific interstitial pneumonia, Organizing pneumonia, Respiratory bronchiolitis, Usual interstitial pneumonia.

Mod Pathol 2002;15(11):1148-1153

Hypersensitivity pneumonitis is a prototype for bronchiolocentric chronic interstitial lung disease with a distinctive histology that is characterized by a lymphocytic bronchiolitis, a patchy interstitial alveolitis with occasional interstitial and airspace

Copyright (C) 2002 by The United States and Canadian Academy of Pathology, Inc.

VOL. 15, NO. 11, P. 1148, 2002 Printed in the U.S.A.

Date of acceptance: August 5, 2002.

Address reprint requests to: Samuel A. Yousem, Department of Pathology,

University of Pittsburgh Medical Center, Presbyterian University Hospital,

Room A610, 200 Lothrop Street, Pittsburgh, PA 15213-2582; e-mail:

yousemsa@msx.upmc.edu; fax: 412-647-3399.

DOI: 10.1097/01.MP.0000037309.04985.B4 plugs of fibromyxoid connective tissue, and poorly formed granulomas (1-3). This triad of morphologic changes allows the pathologist to direct the pulmonologist to question the patient for specific inhalational exposures. We have accumulated 10 cases that have very similar morphologic findings to hypersensitivity pneumonitis, with the exclusion of interstitial granulomas, in which extensive investigations failed to reveal a cause for the inflammation. The clinicopathologic features of this idiopathic bronchiolocentric interstitial pneumonia are the basis of this report which suggests a more aggressive and life threatening biologic behavior for bronchiolocentric interstitial pneumonia than normally associated with hypersensitivity pneumonitis or some of the other conditions in the histologic differential diagnosis.

\section{MATERIALS AND METHODS}

The surgical pathology files of the Department of Pathology of the University of Pittsburgh Medical Center-Presbyterian University Hospital were searched for all cases of unclassified interstitial pneumonia or cases "suggestive of" hypersensitivity pneumonia, diagnosed by open lung biopsy from 1987-2002. Twenty-three cases were characterized by the presence of a bronchiolocentric interstitial pneumonia lacking interstitial epithelioid cell aggregates, interstitial giant cells, or poorly formed non-necrotizing granulomas. Eight cases occurring in patients with known autoimmune disease (five with rheumatoid arthritis, two with Sjögren's syndrome, one with scleroderma) were excluded because their pulmonary disease was most likely related to their underlying rheumatologic condition. Four cases were eliminated because they were likely to be drug-induced (three methotrexate, one amiodarone) and one patient had a history of hard metal exposure.

The remaining 10 patients had their medical records extensively evaluated for allergies and for known causes of hypersensitivity pneumonitis. Questionnaires were completed by referring pulmonologists, and/or patients which specifically 
asked about exposure to birds, window air conditioners, noxious gases, or dusty environments, including work on farms. Each pulmonologist indicated that, as best as possible, they could "confidently exclude the possibility of hypersensitivity pneumonitis from your clinical differential diagnosis." Included in this examination were specific questions with regard to medications with known pulmonary toxicity and other medical and pulmonary conditions that could cause respiratory disease, e.g., bronchiectasis, asthma, aspiration, cigarette smoking.

Pathologic features that were assessed included the following: distribution of the inflammatory infiltrate, presence of a patchy interstitial pneumonitis, acute bronchiolitis, intraluminal fibromyxoid connective tissue plugs, peribronchiolar scarring, muscular hyperplasia of the small airways, peribronchiolar fibrosis, air space organization ("organizing pneumonia"), bronchiolar metaplasia, desquamative interstitial pneumonia-like reactions, lymphoid aggregates, mucostasis, presence of eosinophils and vascular atherosclerosis. As noted previously, the presence of interstitial epithelioid histiocytes, giant cells, and granulomas were exclusionary.

\section{RESULTS}

Ten patients comprised the study group (Table 1). Eight were women (80\%), two were men $(20 \%)$; seven were Caucasian and three were AfricanAmericans. Ages at the time of biopsy ranged from 28-69 years with a mean of 46.7 years and a median of 44.0 years. Patients presented with a history of respiratory complaints that ranged from five to 24 months (mean 10.0 months; median 13 months). The most common complaints were shortness of breath (eight) and dry cough (five) although wheezing, chest pain, and dyspnea on exertion (two each) were also noted. Sputum production and a history of "recurrent pneumonia" was noted in one patient.
In nine patients, symptoms developed insidiously with only one case (Patient 3 ) having a more acute course (5 months).

Chest radiographs showed bibasilar interstitial infiltrates in nine patients with one patient having normal chest radiographs. Two patients had alveolar infiltrates superimposed on the underlying reticular and reticulonodular interstitial disease. Honeycomb lung was not reported in any case. High resolution CT scans or their reports were available for review in only six cases and confirmed the above findings, and excluded large and small airway disease. Pulmonary function tests showed restrictive lung disease with reduced lung volumes (total lung capacity less than $80 \%$ of predicted for age) and abnormalities of carbon monoxide diffusing capacity (less than $80 \%$ of predicted) in six cases. No patient had significant airflow obstruction despite a history of current or previous cigarette smoking in four patients. Two patients did have a previous diagnosis of gastroesophageal reflux disease. One patient had history of a skin biopsy showing "morphea" but lacked the diagnosis of systemic sclerosis. All 10 patients had negative antinuclear antibody studies; four had negative rheumatoid factor. No patient had a history of inflammatory bowel disease.

Followup information was available in nine patients and ranged from 4-108 months (mean 48.2 months). All nine patients received steroid therapy (dosage range $30-60 \mathrm{mg} /$ day) and two received Cyclophosphamide ( $2 \mathrm{mg} / \mathrm{kg} /$ day). Three patients died of pulmonary disease (48, 60, 96 months), five patients were alive with persistent or progressive pulmonary disease and one patient had no evidence of disease at 84 months followup.

\section{Histopathology}

The most striking feature of these 10 cases was the centrilobular and bronchiolocentric nature of

TABLE 1. Clinical Data-Idiopathic Bronchiolocentric Interstitial Pneumonia

\begin{tabular}{|c|c|c|c|c|c|}
\hline Case Number & Age/Race/Sex & $\begin{array}{l}\text { Presenting Signs and } \\
\text { Symptoms }\end{array}$ & Chest Radiographs & $\begin{array}{l}\text { Smoking } \\
\text { History }\end{array}$ & Status \\
\hline 1 & $28 / \mathrm{W} / \mathrm{F}$ & SOB, cough & Interstitial infiltrates & - & DOD, 48 months \\
\hline 2 & $49 / \mathrm{W} / \mathrm{F}$ & $\begin{array}{l}\text { Cough, wheeze, } \\
\text { chest pain }\end{array}$ & Interstitial infiltrates & + & AWSD, 108 months \\
\hline 3 & $41 / \mathrm{B} / \mathrm{M}$ & SOB, chest pain & Interstitial infiltrates & - & NED, 84 months \\
\hline 4 & $65 / \mathrm{W} / \mathrm{F}$ & SOB & Normal & - & DOD, 60 months \\
\hline 5 & $36 / \mathrm{W} / \mathrm{F}$ & SOB, DOE & Interstitial infiltrates & + & AWPD, 13 months \\
\hline 6 & $53 / \mathrm{W} / \mathrm{F}$ & $\begin{array}{l}\text { Cough, recurrent } \\
\text { pneumonia }\end{array}$ & $\begin{array}{l}\text { Interstitial and alveolar } \\
\text { infiltrates }\end{array}$ & - & AWSD, 4 months \\
\hline 7 & $46 / \mathrm{B} / \mathrm{F}$ & SOB, cough & Interstitial infiltrates & + & Lost to F/U \\
\hline 8 & $69 / \mathrm{W} / \mathrm{F}$ & SOB & Interstitial infiltrates & - & DOD, 96 months \\
\hline 9 & $41 / \mathrm{W} / \mathrm{M}$ & $\begin{array}{l}\text { SOB, DOE, cough, } \\
\text { wheeze }\end{array}$ & $\begin{array}{l}\text { Interstitial infiltrates with } \\
\text { focal ground glass change }\end{array}$ & + & AWPD, 9 months \\
\hline 10 & $39 / \mathrm{B} / \mathrm{F}$ & SOB, cough & Interstitial infiltrates & - & AWPD, 12 months \\
\hline
\end{tabular}

W, Caucasian; B, African-American; M, male; F, female; SOB, shortness of breath; DOE, dyspnea on exertion; +, positive; -, negative; NED, no evidence of disease; DOD, dead of disease; AWDS, alive with stable disease; AWPD, alive with progressive disease. 
the chronic inflammatory cell infiltrate (Fig. 1). At low magnification, the small airways were cuffed by an irregular band of lymphocytes and plasma cells ("mild intensity" in eight cases and "marked intensity" in two) with the infiltrate extending into the peribronchiolar alveolar septa and gradually dissipating into the distal interstitium of the acinus (Fig. 2). This resulted in the appearance of a lymphocytic bronchiolitis accompanied by a patchy mild interstitial pneumonitis with relative sparing of the subpleural and peripheral lobular space by the inflammatory infiltrate (Fig. 3). In seven cases, the airway inflammation was accompanied by peribronchiolar fibrosis with co-existent bronchiolar and goblet cell metaplasia ("microscopic honeycomb change") (Fig. 4). In these cases, peribronchiolar alveolar septa were thickened and fibrotic but architectural remodeling of parenchyma was not observed. In five cases rare foci of fibromyxoid tissue were noted beneath the attenuated and flattened bronchiolar mucosa $(<5 \%)$; however, non-epithelialized fibromyxoid plugs were rarely seen in the lumens of the airways. Centrilobular finely pigmented smokers macrophages were noted in three of the four cigarette smokers. Wisps of airway luminal mucus were seen in bronchioles in all nine cases and included

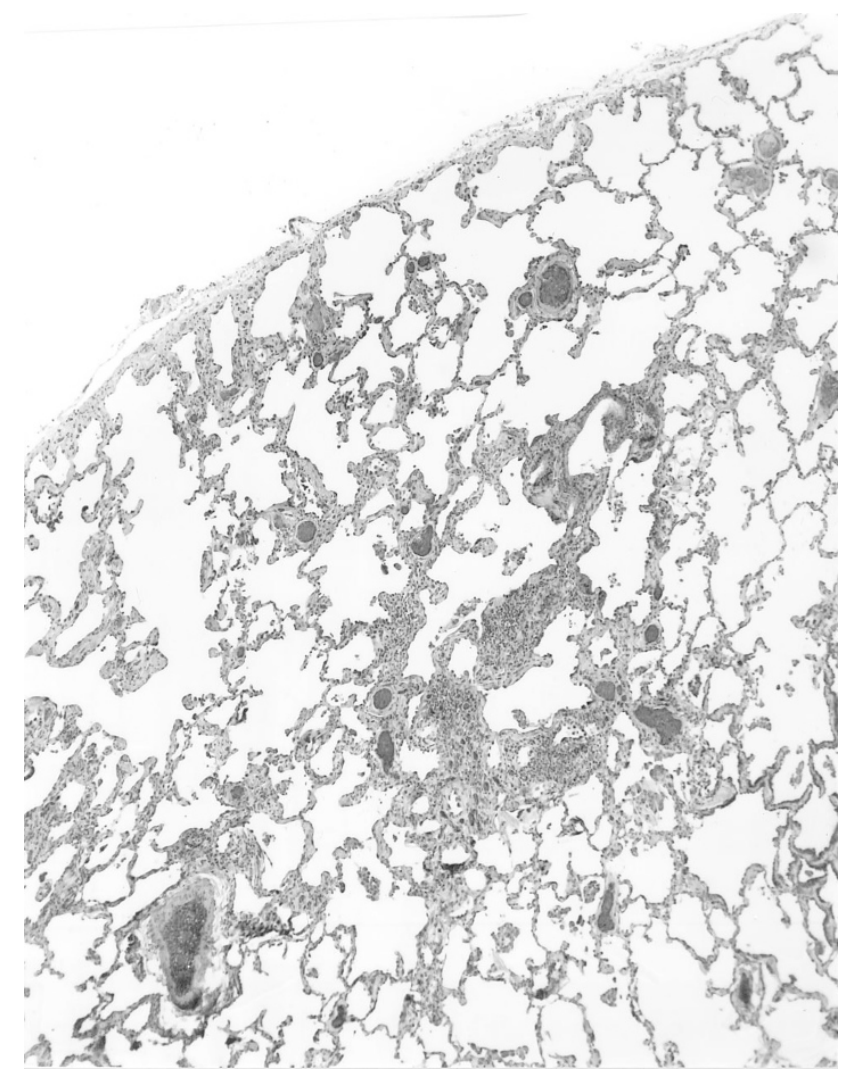

FIGURE 1. Bronchiolocentric interstitial pneumonia. At low magnification, bronchiolocentric interstitial pneumonia is characterized by centrilobular inflammation which dissipates in the distal acinus and subpleural pulmonary parenchyma (H\&E, x15).

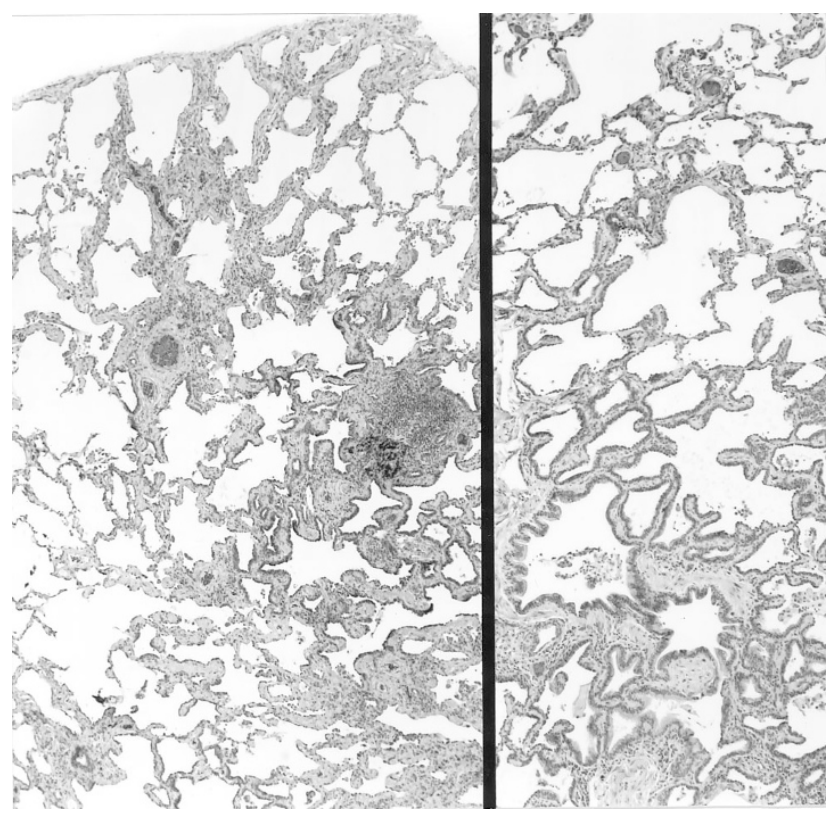

FIGURE 2. Bronchiolocentric interstitial pneumonia. At left, the centrilobular compartment of the lobule shows fibrosis with metaplastic epithelium, in continuity with the bronchioles, as noted at right. Some would consider this change as "microscopic honeycomb" change $(\mathrm{H} \& \mathrm{E}, \times 25$, left $, \mathrm{H} \& \mathrm{E}, \times 40$, right $)$

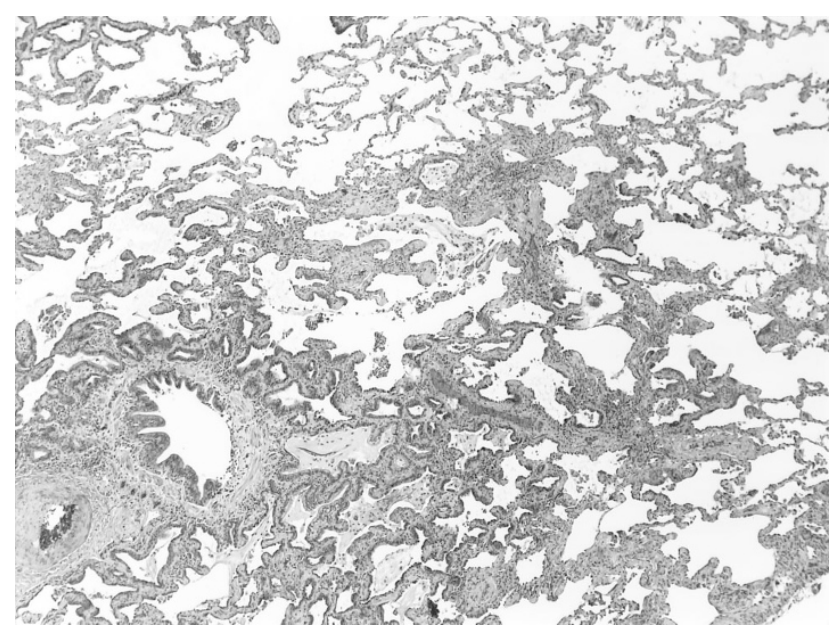

FIGURE 3. Bronchiolocentric interstitial pneumonia. The inflammatory process emanates from the bronchovascular bundle and is accompanied by centrilobular fibrosis and metaplasia. Mucostasis is also prominent along with an interstitial alveolar septal infiltrate. Note that the periphery of the lobule is relatively spared (H\&E, $\mathrm{x} 40)$.

the focal presence of foamy macrophages randomly distributed in lobular airspaces.

The distal portion of the lobule showed a patchy interstitial mononuclear infiltrate (Fig. 5). Rare and infrequent air space granulation tissue polyps were noted in four cases. Peribronchiolar and perivascular lymphoid aggregates were seen in six cases.

Subsidiary histologic findings included the following: apical cap subpleural fibrosis (two cases), bronchiolectasia, emphysematous change, an old thromboembolus and a pulmonary chemodectoma 


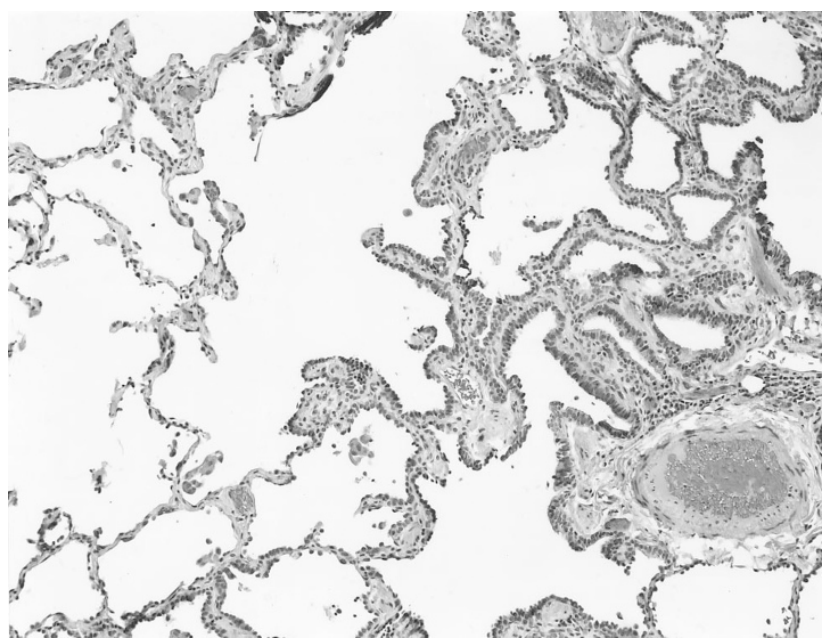

FIGURE 4. Bronchiolocentric interstitial pneumonia. Scarring of the bronchioles was associated with metaplasia of the epithelium with relative sparing of the centrilobular and distal alveolar septa. Although the alveolar skeleton has not been remodeled, some consider this peribronchiolar fibrosis as microscopic honeycombing (H\&E, x200).

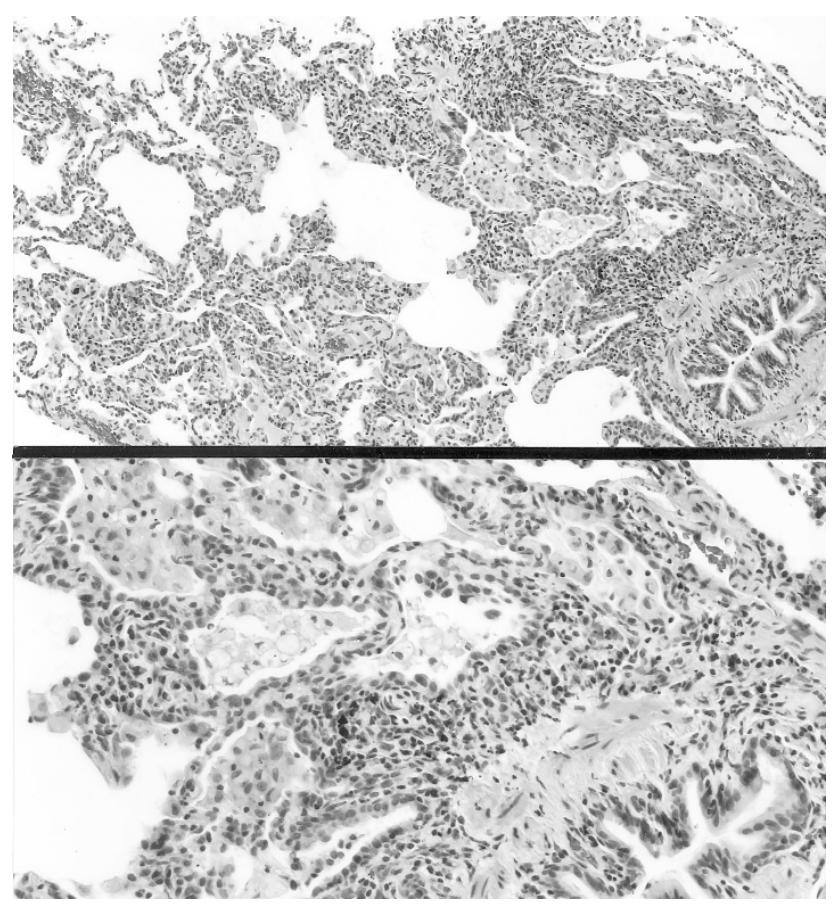

FIGURE 5. Bronchiolocentric interstitial pneumonia. At top, an interstitial mononuclear inflammatory infiltrate involved the bronchovascular bundle and perivascular zones of the distal lobule. The infiltrate consisted of small round lymphocytes and plasma cells and often was associated with air space foamy macrophages at bottom (H\&E, $\times 40$, top; H\&E, $\times 160$, bottom).

(one case each). As noted previously, no evidence of gross honeycomb lung or a primary subpleural distribution of interstitial injury and fibrosis was observed.

No histologic feature correlated with prognosis including peribronchiolar fibrosis or microscopic honeycomb change, although it should be noted that the number of patients in this series is limited.

\section{DISCUSSION}

Idiopathic chronic interstitial pneumonias are inflammatory processes segregated histologically by the distribution of disease in the lung, the lobular compartment primarily affected, the age of maturity of the interstitial fibrosis and the presence of specialized histologic features such as the pattern of air space filling by macrophages or the presence of granulomas (Table 2) (4-6). The patterns of lung inflammation and fibrosis that allow classification of the chronic interstitial pneumonias are usually idiopathic and known causes must be clinically and pathologically excluded. Specifically, autoimmune diseases, drug and allergic reactions, and pneumoconiosis should be investigated and eliminated before placing an interstitial pneumonia in cryptogenic category. Of the idiopathic chronic interstitial pneumonias, only respiratory bronchiolitis associated interstitial lung disease has a predominantly bronchiolocentric distribution $(7,8)$. The 10 cases reported in this series are clinicopathologically distinct from respiratory bronchiolitis associated interstitial lung disease and hypersensitivity pneumonitis and represent a pattern of cryptogenic chronic interstitial lung disease with an airway-centered pattern of inflammation.

Bronchiolocentric interstitial pneumonia is a bronchiolocentric process associated with centrilobular and peribronchiolar fibrosis and a patchy distal lymphocytic alveolitis lacking the presence of interstitial granulomas. A marked predilection for women in bronchiolocentric interstitial pneumonia is unusual among interstitial pneumonias although these patients present with similar respiratory complaints of comparable duration. Radiographs show bilateral predominantly lower lobe interstitial infiltrates. Honeycomb change was not identified in our cases, contrasting with its frequent presence in usual interstitial pneumonia (approximately 80\%) and occasional presence in hypersensitivity pneumonitis and nonspecific interstitial pneumonia (approximately $15 \%)(5,9-13)$. Like these conditions, bronchiolocentric interstitial pneumonia is associated with functional restrictive lung disease with gas exchange abnormalities. Most disturbing in bronchiolocentric interstitial pneumonia, in contrast to respiratory bronchiolitis associated interstitial lung disease and hypersensitivity pneumonitis, is its relatively poor prognosis $(1,14-16)$. At approximately 4 years mean followup, 33\% of the patients had died of disease and $56 \%$ of patients had persistent or progressive complaints. This prognosis is significantly worse than usual interstitial pneumonia and closer to that of fibrotic nonspecific interstitial pneumonia, but our series is too small to be definitive $(5,6,10,15,16)$. 
TABLE 2. Major Differential Diagnoses for BrIP

\begin{tabular}{|c|c|c|c|c|c|}
\hline $\begin{array}{l}\text { Idiopathic Chronic } \\
\text { Interstitial Pneumonia }\end{array}$ & Distribution & $\begin{array}{l}\text { Primary Lobular Site } \\
\text { of Injury }\end{array}$ & $\begin{array}{l}\text { Fibroblastic } \\
\text { Foci }\end{array}$ & Special Reactions & Prognosis \\
\hline UIP & Patchy & Subpleural/paraseptal & ++ & - & Poor \\
\hline NSIP & Diffuse & Alveolar septal & $+($ rare $)$ & - & $\begin{array}{l}\text { Good-except } \\
\text { fibrotic phase }\end{array}$ \\
\hline DIP & Diffuse & Alveolar septal & $+($ rare $)$ & $\begin{array}{r}\text { Diffuse airspace } \\
\text { macrophages }\end{array}$ & Good \\
\hline RB/ILD & Patchy & Bronchiolocentric & - & $\begin{array}{c}\text { Centrilobular airspace } \\
\text { macrophages }\end{array}$ & Good \\
\hline BOOP & Patchy & Bronchiolocentric & $\begin{array}{c}+++ \\
\text { (airspace and intraluminal) }\end{array}$ & - & Good \\
\hline BrIP & Patchy & Bronchiolocentric & + & $\begin{array}{l}\text { Peribronchiolar } \\
\text { scarring }\end{array}$ & Fair \\
\hline
\end{tabular}

UIP, usual interstitial pneumonia; NSIP, nonspecific interstitial pneumonia; DIP, desquamative interstitial pneumonia; RB/ILD, respiratory bronchiolitis associated interstitial lung disease; BOOP/COP, bronchiolitis obliterans organizing pneumonia (note: some pathologists do not include BOOP as a chronic interstitial pneumonia).

- , not present; +-+++ present; intensity scale $1-3+$.

Bronchiolocentric interstitial pneumonia has histologic similarity to hypersensitivity pneumonitis although the degree of centrilobular fibrosis appears more extensive and granulomas are absent. In the study of hypersensitivity pneumonitis by Coleman et al., cases of hypersensitivity pneumonitis were defined by the mandatory presence of granulomas (1). It is possible that bronchiolocentric interstitial pneumonia represents a form of chronic hypersensitivity pneumonitis for which the inhalational stimulus has not been identified despite our extensive workup for this condition. Furthermore granulomas are not always present in hypersensitivity pneumonitis, being described in between $60-80 \%$ of known cases $(3,9$, 17-21). Coleman et al. did report, however, that the more aggressive cases of hypersensitivity pneumonitis in their study were those in which an underlying antigen was not identified and it may be that these cases fall into the idiopathic bronchiolocentric interstitial pneumonia cases described herein. Even if bronchiolocentric interstitial pneumonia was a form of hypersensitivity pneumonitis, however, there are some distinguishing features including the predilection for women, the degree of centrilobular scarring, and the poor prognosis. In studies of pigeon breeder's lung, 4 year survivals of better than $80 \%$ are expected, in contrast to the results of our small series of bronchiolocentric interstitial pneumonia (19). It is important to emphasize that bronchiolocentric interstitial pneumonia may also represent a late stage of a preexisting inflammatory process such as bronchiolitis obliterans organizing pressure, healing infectious pneumonia, nonspecific interstitial pneumonia, or others. Only larger studies evaluating the pattern of bronchiolocentric interstitial pneumonia described herein will be able to answer its relationship to these conditions, and we suspect that many of these cases will be cryptogenic as we have described.

From a morphologic perspective, bronchiolocentric interstitial pneumonia needs to be separated from bronchiolitis (12). The presence of a female predilec- tion, restrictive physiology, and infiltrative lung disease on radiographs would be clinical discriminators. On histologic grounds, the amount of alveolar septal infiltration of the distal lobule would be unusual for chronic airway disease. Bronchiectasis needs to be considered as well although sputum production was not a clinical component and chest radiographs failed to show ectasia of airways but instead demonstrated interstitial infiltrates. Nonspecific interstitial pneumonia is a major differential diagnostic consideration. Katzenstein et al. (14) reported 14/31 cases of nonspecific interstitial pneumonia with a bronchiolocentric distribution of inflammation; however, the remaining cases demonstrated a diffuse alveolar septal infiltrate. Travis et al. (6) specifically excluded a bronchiolocentric distribution in their description of cases of nonspecific interstitial pneumonia. A current working group on nonspecific interstitial pneumonia sponsored by the American Thoracic Society has also specifically excluded bronchiolocentric interstitial injury from its definition of nonspecific interstitial pneumonia (Thomas V. Colby, MD, personal communication). The histologic pattern of disease, predilection for women, and aggressive clinical course (more than $85 \%$ of patients DOD or AWPD, Table 1) also contrasts with the prognosis of nonspecific interstitial pneumonia. Nonetheless, time may show that bronchiolocentric interstitial pneumonia represents a subset of NSIP. Respiratory bronchiolitis associated interstitial lung disease also needs to be distinguished from bronchiolocentric interstitial pneumonia. As noted previously, the clinicopathologic setting is different in that bronchiolocentric interstitial pneumonia occurs primarily in women and in $60 \%$ of our cases, bronchiolocentric interstitial pneumonia patients were non-smokers. Respiratory bronchiolitis tends to have a very minimal peribronchiolar inflammatory infiltrate and peribronchiolar fibrosis, with a paucity of distal interstitial acinar involvement. Prominent centrilobular smoker's macrophages are defini- 
tional for respiratory bronchiolitis associated interstitial lung disease.

Like most interstitial inflammatory processes, the idiopathic form of bronchiolocentric interstitial pneumonia must be separated from similar patterns of lung injury that have known causes or associations as noted in our Materials and Methods. A bronchiolocentric interstitial pneumonia may be a manifestation of autoimmune disease $(5,14)$. Rheumatoid arthritis, Sjögren's syndrome, and scleroderma may all have a similar pattern of lung infiltration. Drug induced pulmonary injury, particularly that caused by methotrexate and amiodarone, can display bronchiolocentric inflammation (22). Aspiration induced pulmonary disease is bronchiolocentric and cicatricial but usually lacks lobular interstitial inflammation while the presence of foreign material and often eosinophils can be helpful discriminators. Pulmonary Langerhans cell histiocytosis has airway-centered nodules of Langerhans cells and could conceivably be confused with bronchiolocentric interstitial pneumonia in its early or very late stages although the alveolar septal inflammation would be atypical. Inhalational lung disease, both pneumoconiosis and toxic gas exposure should always be considered in any airway centered inflammatory processes. Asbestosis and hard metal pneumoconiosis have centrilobular injury early in their disease course and may be associated with an interstitial pneumonitis. In all cases of bronchiolocentric interstitial pneumonia, a careful clinical history, special stains e.g., iron and observation of distinctive histologic features e.g., cannibalistic giant cells, are helpful in excluding occupational lung disease.

In summary, idiopathic bronchiolocentric interstitial pneumonia appears to represent a pattern of chronic interstitial lung disease with a distinct histopathology that is reminiscent of hypersensitivity pneumonitis. In the absence of interstitial granulomas and a history of exposure to offending antigens, idiopathic bronchiolocentric interstitial pneumonia should be considered as an alternative to hypersensitivity pneumonitis or nonspecific interstitial pneumonia. Its predilection for women, distinctive histology including the absence of granulomas and the presence of peribronchiolar fibrosis, and the relatively poor prognosis, suggest that bronchiolocentric interstitial pneumonia should be considered in the classification of idiopathic chronic interstitial pneumonias. Studies beyond this report will hopefully expand the clinicopathologic characteristics of this disease and confirm these initial observations.

Acknowledgments: The authors acknowledge the secretarial assistance of Diana Winters and the photographic expertise of Linda Shab.

\section{REFERENCES}

1. Coleman A, Colby TV. Histologic diagnosis of extrinsic allergic alveolitis. Am J Surg Pathol 1988;12:514-8.

2. Reyes CN, Wenzel FJ, Lawton BR, et al. The pulmonary pathology of farmer's lung disease. Chest 1982;81:142-6.

3. Seal RME, Hapke EJ, Thomas GO, et al. The pathology of the acute and chronic stages of farmer's lung. Thorax 1968;23:469-89.

4. American Thoracic Society and European Respiratory Society. Idiopathic pulmonary fibrosis: diagnosis and treatment. International consensus statement. Am J Respir Crit Care Med 2000;161:646-64.

5. Katzenstein ALA, Myers JL. Idiopathic Pulmonary fibrosis: clinical relevance of pathologic classification. Am J Respir Crit Care Med 1998;157:1301-15.

6. Travis WD, Matsui K, Moss J, et al. Idiopathic nonspecific interstitial pneumonia: prognostic significance of cellular and fibrosing patterns. Survival comparison with UIP and desquamative interstitial pneumonia. Am J Surg Pathol 2000;24:19-33.

7. Moon J, DuBois RM, Colby TV, et al. Clinical significance of respiratory bronchiolitis on open lung biopsy and its relationship to smoking related interstitial lung disease. Thorax 1999;54:1009-14.

8. Yousem SA, Colby TV, Gaensler EA. Respiratory bronchiolitis-associated interstitial lung disease and its relationship to desquamative interstitial pneumonia. Mayo Clin Proc 1989;64:1373-80.

9. Ando M, Arima K, Yoneda R, et al. Japanese summer-type hypersensitivity pneumonitis: geographic distribution, home environment, and clinical characteristics of 621 cases. Am Rev Respir Dis 1991;144:765-9.

10. Carrington CB, Gaensler EA, Coutu RE, et al. Natural history and treated course of usual and desquamative interstitial pneumonia. N Engl J Med 1978;298:801-9.

11. Gross TJ, Hunninghake GW. Idiopathic pulmonary fibrosis. N Engl J Med 2001;345:517-25.

12. Hansell DM, Moskovic E. High-resolution computed tomography in extrinsic allergic alveolitis. Clin Radiol 1991;43:8-12.

13. Katzenstein ALA, Fiorelli RF. Nonspecific interstitial pneumonia/fibrosis: histologic features and clinical significance. Am J Surg Pathol 1994;18:136-47.

14. Katzenstein ALA, Myers JL. Nonspecific interstitial pneumonia and the other idiopathic interstitial pneumonias: classification and diagnostic criteria. Am J Surg Pathol 2000;24:1-3.

15. Nagai S, Kitaichi $\mathrm{M}$, Itoh $\mathrm{H}$, et al. Idiopathic nonspecific interstitial pneumonia/fibrosis: comparison with idiopathic pulmonary fibrosis and BOOP. Eur Respir J 1998;12:1010-19.

16. Nicholson AG, Colby TV, Dubois RM, et al. The prognostic significance of the histologic pattern of interstitial pneumonia in patients presenting with the clinical entity of cryptogenic fibrosing alveolitis. Am J Respir Crit Care Med 2000;162:2213-7.

17. Emanuel DA, Wenzel FJ, Bowerman CI, et al. Farmer's lung: clinical, pathologic and immunologic study of twenty-four patients. Am J Med 1964;37:392-9.

18. Perez-Padilla R, Gaxiola M, Salas J, et al. Bronchiolitis in chronic pigeon breeder's disease. Morphologic evidence of a spectrum of small airway lesions in hypersensitivity pneumonitis induced by avian antigens. Chest 1996;110:371-7.

19. Perez-Padilla R, Salas J, Chapela R, et al. Mortality in Mexican patients with chronic pigeon breeder's lung compared with those withUIP. Am Rev Respir Dis 1993;148:49-53.

20. Salvaggio JE. Extrinsic allergic alveolitis (hypersensitivity pneumonitis): past, present, and future. Clin Exp Allergy Suppl 1997;27:18-25.

21. Selman M, Vargas MH. Airway involvement in hypersensitivity pneumonitis. Curr Opin Pulm Med 1998;4:9-15.

22. Imokawa S, Colby TV, Leslie KO, et al. Methotrexate pneumonitis: review of the literature and histopathological findings in nine patients. Eur Respir J 2000;15:373-81. 